\title{
A log derivative formulation of reaction rate theory
}

\author{
David E. Manolopoulos \\ Department of Chemistry, University of Nottingham, Nottingham NG7 2RD, UK
}

and

John C. Light

Department of Chemistry and The James Franck Institute, University of Chicago, Chicago, IL 60637, USA

Received 27 September 1993; in final form 21 October 1993

\begin{abstract}
The reaction rate theory of Miller, Schwartz and Tromp is reformulated using a complex Bloch boundary value operator to enforce the scattering boundary conditions. This Bloch operator requires a knowledge of the log derivative of the outgoing wavefunction on the boundary of the interaction region, and this in turn can be approximated semiclassically from a knowledge of the interaction potential on the boundary. The resulting absorbing $\log$ derivative boundary conditions are shown to work well in practice, reducing the range over which the quantum-mechanical problem has to be solved to a narrow region enclosing the relevant turning points. For example they are shown to be at least three times more effective in reducing the required size of the interaction region for a standard barrier tunnelling problem than more conventional absorbing potentials.
\end{abstract}

\section{Introduction}

The cumulative reaction probability $N(E)$ for a chemical reaction is defined as the sum of all stateto-state reaction probabilities at energy $E[1]$ and is related to the thermal rate constant $k(T)$ by

$k(T)=\left[2 \pi h Q_{\mathrm{r}}(T)\right]^{-1} \int_{-\infty}^{+\infty} \mathrm{e}^{-E / k T} N(E) \mathrm{d} E$,

where $Q_{\mathrm{r}}(T)$ is the partition function per unit volume of the reactants. One obvious way to calculate $N(E)$ is thus to perform a converged state-to-state quantum reactive scattering calculation at each energy, and then simply add the state-to-state reactive probabilities. However this is not an efficient way to proceed if $N(E)$ (or $k(T)$ ) is the primary quantity of interest, because a converged state-to-state reactive scattering calculation requires the use of a basis set (or the equivalent) which extends right out to the asymptotic reactant and product regions. The success of both classical [2] and semiclassical [3] transition state theories in their respective domains suggests that one should be able instead to perform a quantum-mechanical calculation of $N(E)$ in a more confined interaction region which only contains the relevant reactive dynamics, and the search for practical ways to do this has been a major goal of theoretical reaction dynamics for several years [4].

The reaction rate theory of Miller, Schwartz and Tromp [5] is a step in the right direction. The key observation in this theory is that $N(E)$ can be written as an operator trace which is independent of the basis in which it is evaluated, with the consequence and the asymptotic reactant and product quantum states do not have to be used as basis functions and can be eliminated from the calculation. Moreover the new basis that is introduced to evaluate the trace can, at least in principle, be confined to the physically relevant interaction region of the reaction.

The elegance of this formulation tends to obscure an important point, however, which is that in eliminating the reactant and product quantum states one is also eliminating the state-to-state boundary conditions. These scattering boundary conditions are an essential part of the problem which must somehow 
be re-introduced, but they cannot be re-introduced exactly without solving the Schrödinger equation outside the interaction region. Thus a genuine practical implementation of the Miller-Schwartz-Tromp theory which only solves the Schrödinger equation within a confined interaction region must inevitably approximate the scattering boundary conditions.

It is probably fair to say that this difficulty with the scattering boundary conditions is the main reason why the Miller-Schwartz-Tromp theory has only been used in comparatively few practical reaction rate calculations to date. In fact most of the applications that have appeared have been based on the time-dependent flux correlation function version of the theory [5], which focuses on $k(T)$ rather than $N(E)$. Here the usual approach to the scattering boundary conditions is to use an ordinary basis set representation of the interaction region in which the boundary conditions are applied incorrectly, but to make the interaction region large enough so that the resulting unphysical boundary reflections are postponed until after the physical reactive dynamics has been calculated [6-9]. This approach has in fact been used successfully in some fully three-dimensional calculations $[7,8]$. However it is clearly only viable for direct reactions without long-lived collision complexes, and it is difficult to implement reliably even for these [4].

A more promising approach is to try to apply the boundary conditions correctly. One way to do this is to introduce an artificial (optical) absorbing potential to enforce the outgoing-wave scattering boundary conditions, as proposed by Neuhauser and Baer [10]. Seideman and Miller have recently adapted this idea in their use of coordinate-dependent absorbing potentials for the time-independent evaluation of $N(E)$ [11,12]. The resulting scheme is very impressive [12], and suggests that the full potential of the theory for calculating cumulative reaction probabilities and thermal rate constants even for polyatomic chemical reactions may soon be realised.

In time-dependent calculations such as those of Neuhauser and Baer a range of energies is present in a single wavepacket, and it is necessary to use an energy-independent optical potential which imposes boundary conditions on the entire wavepacket. For the time-independent calculation of $N(E)$, however, the boundary conditions can be specified at each en- ergy. The aim of the present Letter, is to show how such energy-dependent scattering boundary conditions can be re-introduced into the Miller-SchwartzTromp theory with the help of a complex Bloch boundary value operator, and to argue that this may offer formal as well as practical advantages for future reaction rate calculations.

\section{Reaction rate theory}

The expression for $N(E)$ that was derived by Miller, Schwartz and Tromp consists of a trace of an operator product involving flux and microcanonical density operators which does not involve any explicit reference to the asymptotic reactant and product quantum states [5],

$N(E)=\frac{1}{2}(2 \pi \hbar)^{2} \operatorname{tr}\{\delta(E-H) F \delta(E-H) F\}$,

where

$\delta(E-H)=-\frac{1}{\pi} \operatorname{Im}\left[G^{+}(E)\right]$,

with

$G^{+}(E)=\lim _{\epsilon \rightarrow 0}(E+\mathrm{i} \epsilon-H)^{-1}$,

and

$F=\frac{\mathrm{i}}{\hbar}[H, h(f(q))]$.

Here the density operator $\delta(E-H)$ has been written in terms of the imaginary part of the scattering Green function $G^{+}(E)$ to facilitate the following discussion. The flux operator $F$ involves the commutator of the Hamiltonian $H$ with a Heaviside step function $h(f(q))$, where $f(q)$ is defined such that the equation $f(q)=0$ is a dividing surface between reactants and products in coordinate space which leaves the reactants in $f(q)<0$ and the products in $f(q)>0$ [5]. Although eq. (1) is exact for any such choice of dividing surface it is usual (and expedient) in practice to choose a surface which passes through the physically relevant interaction region of the reaction [4].

In order to make these equations less abstract we shall focus throughout this Letter on the simplest possible model for a reaction, namely the penetration of a one-dimensional potential barrier along a 
reaction coordinate q. The Hamiltonian for this model is

$H=-\frac{\hbar^{2}}{2 m} \frac{\mathrm{d}^{2}}{\mathrm{~d} q^{2}}+V(q)$,

and the cumulative reaction probability $N(E)$ is simply the barrier transmission probability as a function of the energy $E$. The dividing surface between reactants $(q \rightarrow-\infty)$ and products $(q \rightarrow+\infty)$ is taken to be $q=0$, so that the flux operator in eq. (4) reduces to

$F=\frac{\mathrm{i}}{\hbar}[H, h(q)]$.

These restrictions do not imply any real loss of generality, and we shall give references to where more general expressions can be found at each stage in the following argument.

The key thing that is required to get a practical scheme for calculating $N(E)$ from the formulation in eqs. (1) to (4), as discussed in the introduction, is a recipe for approximating the outgoing-wave scattering boundary conditions implied by the $+i \epsilon$ in eq. (3). Ideally, one would like to approximate these boundary conditions in such a way that the trace in eq. (1) can be evaluated in a small basis which spans a correspondingly small physically relevant interaction region enclosing the dividing surface. With this end in mind Seideman and Miller suggest the prescription [11,12]

$+i \epsilon \rightarrow+i \epsilon(q)$,

where $i \epsilon(q)$ is a coordinate-dependent optical potential which is zero (or nearly zero) in the interaction region and "turns on" in some predetermined manner at the edges. This prescription is simple to implement, appears to work well in practice, and has been used in some impressive calculations [12]. However it is a purely empirical rather than a rigorously derived approximation to the true $+i \epsilon$ (i.e. to the true outgoing-wave scattering boundary conditions), and has features such as the arbitrary choice of functional form for $\epsilon(q)$ which it would clearly be preferable to avoid.

An alternative way to construct the outgoing-wave scattering Green function $G^{+}(E)$ within a finite interval $q_{\mathrm{r}} \leqslant q \leqslant q_{\mathrm{p}}$ has in fact been available for some time $[13,14]$. In the present context this construction boils down to the prescription

i $\epsilon \rightarrow-\mathscr{L}(E)$,

where $\mathscr{L}(E)$ is the complex Bloch boundary value operator [13] \#1

$$
\begin{aligned}
& \mathscr{L}(E)=\frac{\hbar^{2}}{2 m}\left[\delta\left(q-q_{\mathrm{p}}\right)\left(\frac{\mathrm{d}}{\mathrm{d} q}-L_{\mathrm{p}}^{+}\left(q_{\mathrm{p}}\right)\right)\right. \\
& \left.-\delta\left(q-q_{\mathrm{r}}\right)\left(\frac{\mathrm{d}}{\mathrm{d} q}-L_{\mathrm{r}}^{+}\left(q_{\mathrm{r}}\right)\right)\right],
\end{aligned}
$$

and $L_{\mathrm{r}}^{+}\left(q_{\mathrm{r}}\right)$ and $L_{\mathrm{p}}^{+}\left(q_{\mathrm{p}}\right)$ are the energy-dependent $\log$ derivatives of outgoing scattering wavefunctions $\psi_{\mathrm{r}}^{+}(q)$ and $\psi_{\mathrm{p}}^{+}(q)$ evaluated on the two boundaries,

$L_{\mathrm{r}}^{+}\left(q_{\mathrm{r}}\right)=\left(\frac{\mathrm{d} \psi_{\mathrm{r}}^{+}(q)}{\mathrm{d} q}\right)_{q_{\mathrm{r}}} \psi_{\mathrm{r}}^{+}\left(q_{\mathrm{r}}\right)^{-1}$,

and

$L_{\mathrm{p}}^{+}\left(q_{\mathrm{p}}\right)=\left(\frac{\mathrm{d} \psi_{\mathrm{p}}^{+}(q)}{\mathrm{d} q}\right)_{q_{\mathrm{p}}} \psi_{\mathrm{p}}^{+}\left(q_{\mathrm{p}}\right)^{-1}$

This prescription has typically been used in the past to construct the Green function $G^{+}(E)$ within an interval $q_{\mathrm{r}} \leqslant q \leqslant q_{\mathrm{p}}$, which extends from the asymptotic reactant region $q_{\mathrm{r}} \ll 0$ to the asymptotic product region $q_{\mathrm{p}} \gg 0$. However, we can avoid this by noting that eq. (8) remains valid for any interval $q_{\mathrm{r}} \leqslant q \leqslant q_{\mathrm{p}}$ provided $L_{\mathrm{r}}^{+}\left(q_{\mathrm{r}}\right)$ and $L_{\mathrm{p}}^{+}\left(q_{\mathrm{p}}\right)$ are evaluated accordingly. At first this might seem like a circular argument, because the exact evaluation of these outgoing-wave log derivatives for a general interval $q_{\mathrm{r}} \leqslant q \leqslant q_{\mathrm{p}}$ requires the solution of the Schrödinger equation outside the interval. However, as we shall see below, $L_{\mathrm{r}}^{+}\left(q_{\mathrm{r}}\right)$ and $L_{\mathrm{p}}^{+}\left(q_{\mathrm{p}}\right)$ are amenable to accurate semiclassical approximations which only depend on the energy $E$ and the interaction potential $V(q)$ and its first derivative $V^{\prime}(q)$ at the boundaries $q_{\mathrm{r}}$ and $q_{\mathrm{p}}$. Thus the interval within which $G^{+}(E)$ is

*1 More precisely, as discussed in the review by Lane and Thomas [14], the delta functions in eq. (9) should be replaced by $\delta\left(q-q_{\mathrm{r}}\right) \rightarrow \lim _{\nu \rightarrow 0} \delta\left(q-\nu-q_{\mathrm{r}}\right) \quad$ and $\delta\left(q-q_{\mathrm{p}}\right) \rightarrow$ $\lim _{v \rightarrow 0} \delta\left(q+\nu-q_{\mathrm{p}}\right)$, with the understanding that the limit be taken after the integration that arises when calculating a matrix element of the operator. These replacements are used in obtaining matrix elements of $\mathscr{L}(E)$ in a basis which spans the interval $q_{\mathrm{r}} \leqslant q \leqslant q_{\mathrm{p}}$ in section 4 below. 
constructed via eqs. (3) and (8), and the problem is subsequently solved via eq. (1), can in practice be reduced to a physically relevant interaction region $q_{\mathrm{r}} \leqslant q \leqslant q_{\mathrm{p}}$ which encloses the dividing surface $q=0$.

The only remaining task in this section is to combine eq. (8) with eqs. (1) to (4) to obtain a computationally convenient expression for $N(E)$, and this is easily accomplished using the manipulations employed by Seidemann and Miller [12] in connection with their prescription for $+i \epsilon$ in eq. (7). The result of these manipulations, which are based here on the observation that both the energy $E$ and the Bloch operator $\mathscr{L}(E)$ commute with the step functions $h(q)$ and $h(-q)=1-h(q)$, is that the expression for $N(E)$ in eq. (1) can be reduced to

$N(E)=4 \operatorname{tr}\left\{\mathscr{L}_{\mathrm{p}} G^{+}(E) * \mathscr{L}_{\mathrm{r}} G^{+}(E)\right\}$,

where

$G^{+}(E)=[E-\mathscr{L}(E)-H]^{-1}$,

and

$$
\begin{aligned}
\mathscr{L}_{\mathrm{r}} & =h(-q) \operatorname{Im}[\mathscr{L}(E)]=+\frac{\hbar^{2}}{2 m} \delta\left(q-q_{\mathrm{r}}\right) \\
& \times \operatorname{Im}\left[L_{\mathrm{r}}^{+}\left(q_{\mathrm{r}}\right)\right]
\end{aligned}
$$

and

$$
\begin{aligned}
\mathscr{L}_{\mathrm{p}}=h(+q) \operatorname{Im}[\mathscr{L}(E)]=-\frac{\hbar^{2}}{2 m} \delta\left(q-q_{\mathrm{p}}\right) \\
\quad \times \operatorname{Im}\left[L_{\mathrm{p}}^{+}\left(q_{\mathrm{p}}\right)\right],
\end{aligned}
$$

and the flux operator $F$ has disappeared. We shall see in section 4 below that this new expression for $N(E)$ has a convenient form for computation when it is combined with basis set representations of the operators $\mathscr{L}_{\mathrm{r}}, \mathscr{L}_{\mathrm{p}}$ and $G^{+}(E)$ in the interaction region $q_{\mathrm{r}} \leqslant q \leqslant q_{\mathrm{p}}$.

Because eq. (12) is an operator trace it is as general as eq. (1), and is not confined to the specific barrier penetration problem considered in eq. (5). However, in order to be able to use eq. (12) in more general situations we need to know how to construct the appropriate Bloch operator $\mathscr{L}(E)$, and this has only been given here (in eq. (9)) for this problem. An appropriate generalisation that is valid when the reactant and product channels are uncoupled outside the interaction region is given in Bloch's paper [13], and this generalisation is itself easy to extend to the (more important) case in which there is residual inelastic coupling within the reactant and product arrangements outside a reactive interaction region. (A key feature of these generalisations is that the multichannel Bloch operator can be written in terms of a basis which diagonalises the Hamiltonian on the boundary of the interaction region, and does not therefore require the re-introduction of asymptotic reactant and product channel eigenfunctions.)

\section{Absorbing log derivative boundary conditions}

Semiclassical approximations to $L_{\mathrm{r}}^{+}\left(q_{\mathrm{r}}\right)$ and $L_{\mathrm{p}}^{+}\left(q_{\mathrm{p}}\right)$ can be obtained directly from the standard (first-order) WKB expressions for outgoing scattering wavefunctions at the reactant and product boundaries. These expressions are [15]

$\psi_{\mathrm{r}}^{+}(q)=k(q)^{-1 / 2} \exp \left(-\mathrm{i} \int_{q_{\mathrm{r}}}^{q} k\left(q^{\prime}\right) \mathrm{d} q^{\prime}\right) C_{\mathrm{r}}^{+}$,

and

$\psi_{\mathrm{p}}^{+}(q)=k(q)^{-1 / 2} \exp \left(+\mathrm{i} \int_{q_{\mathrm{p}}}^{q} k\left(q^{\prime}\right) \mathrm{d} q^{\prime}\right) C_{\mathrm{p}}^{+}$,

where

$k(q)=\{2 m[E-V(q)]\}^{1 / 2 / \hbar}$,

and $C_{\mathrm{r}}^{+}$and $C_{\mathrm{p}}^{+}$are complex constants which fix the normalisations and asymptotic phases of $\psi_{\mathrm{r}}^{+}(q \rightarrow-\infty)$ and $\psi_{\mathrm{p}}^{+}(q \rightarrow+\infty)$. These equations assume that $q_{\mathrm{r}}$ and $q_{\mathrm{p}}$ lie in classically allowed regions, which is clearly also a physical requirement if the interval $q_{\mathrm{r}} \leqslant q \leqslant q_{\mathrm{p}}$ is to be a valid interaction region for the calculation of the barrier transmission probability $N(E)$.

The corresponding first-order WKB approximations to $L_{\mathrm{r}}^{+}\left(q_{\mathrm{r}}\right)$ and $L_{\mathrm{p}}^{+}\left(q_{\mathrm{p}}\right)$ follow from eqs. (10) and (11) as

$L_{\mathrm{r}}^{+}\left(q_{\mathrm{r}}\right)=-\mathrm{i} k\left(q_{\mathrm{r}}\right)+\frac{V^{\prime}\left(q_{\mathrm{r}}\right)}{4\left[E-V\left(q_{\mathrm{r}}\right)\right]}$,

and

$L_{\mathrm{p}}^{+}\left(q_{\mathrm{p}}\right)=+\mathrm{i} k\left(q_{\mathrm{p}}\right)+\frac{V^{\prime}\left(q_{\mathrm{p}}\right)}{4\left[E-V\left(q_{\mathrm{p}}\right)\right]}$, 
where the first term is the simple zero-order WKB approximation in each case. The most important thing to notice about these approximations is that the constants $C_{\mathrm{r}}^{+}$and $C_{\mathrm{p}}^{+}$of eqs. (16) and (17) have cancelled out from eqs. (19) and (20). Thus the outgoing-wave $\log$ derivatives $L_{\mathrm{r}}^{+}\left(q_{\mathrm{r}}\right)$ and $L_{\mathrm{p}}^{+}\left(q_{\mathrm{p}}\right)$ can be approximated directly from a knowledge of the interaction potential $V(q)$ and its first derivative $V^{\prime}(q)$ at the boundaries $q=q_{\mathrm{r}}$ and $q_{\mathrm{p}}$ without invoking any information about the asymptotic reactant and product regions. This makes the approximations useful as absorbing log derivative boundary conditions for the calculation of $N(E)$ in eq. (12).

Note also that these approximations are derived rather than empirical: they are valid when the WKB equations (16) and (17) are accurate, which is basically when the second terms on the right-hand sides of eqs. (19) and (20) are small in absolute magnitude compared with the first [15]. They also become exact when the interaction potential $V(q)$ is constant beyond the two boundaries, which is the case asymptotically for both of the model barrier penetration problems considered in section 5 below.

Eqs. (19) and (20) can be generalised in various ways. It is straightforward to go beyond the first-order WKB approximation, for example, and on doing so one finds that the $n$th order approximations to $L_{\mathrm{r}}^{+}\left(q_{\mathrm{r}}\right)$ and $L_{\mathrm{p}}^{+}\left(q_{\mathrm{p}}\right)$ only depend on $V(q)$ and its first $n$ derivatives at $q_{\mathrm{r}}$ and $q_{\mathrm{p}}$. However, since the first-order approximations in eqs. (19) and (20) already work very well, as shown in section 5 below, these higher-order approximations are unlikely to be necessary in practice. A potentially more useful generalisation is the multichannel WKB method described by Johnson [16], which shows (although Johnson does not give the result explicitly) how eqs. (19) and (20) can be generalised to multichannel situations in which $V(q)$ and $V^{\prime}(q)$ are non-commuting matrices. This generalisation retains the convenient form of eqs. (19) and (20), with the multichannel outgoing-wave log derivative matrices $L_{\mathrm{r}}^{+}\left(q_{\mathrm{r}}\right)$ and $L_{\mathrm{p}}^{+}\left(q_{\mathrm{p}}\right)$ being given solely in terms of $V(q)$ and $V^{\prime}(q)$ at $q=q_{\mathrm{r}}$ and $q_{\mathrm{p}}$. Thus it is again unnecessary to solve the (multichannel) Schrödinger equation outside the interaction region. (Uniform semiclassical approximations to $L_{\mathrm{r}}^{+}\left(q_{\mathrm{r}}\right)$ and $L_{\mathrm{p}}^{+}\left(q_{\mathrm{p}}\right)$ can also be derived very easily in the present context [15], but it is unlikely that they will be so easy to generalise to multichannel problems.)

\section{Basis set representations}

The final stage in the present theory is to form basis set representations of the operators $\mathscr{L}_{\mathrm{r}}, \mathscr{L}_{\mathrm{p}}$ and $G^{+}(E)$ appearing in the expression for $N(E)$ in eq. (12). This is most conveniently accomplished by introducing a set of $n$ real basis functions $\left\{\phi_{i}(q)\right\}_{1}^{n}$ which are orthonormal with respect to integration over the interaction region $q_{\mathrm{r}} \leqslant q \leqslant q_{\mathrm{p}}$ and complete at the boundaries $q=q_{\mathrm{r}}$ and $q_{\mathrm{p}}$. In other words the basis set is constrained to satisfy

$$
\langle i \mid j\rangle=\int_{q_{\mathrm{r}}}^{q_{\mathrm{p}}} \phi_{i}(q) \phi_{j}(q) \mathrm{d} q=\delta_{i j},
$$

for $i, j=1,2, \ldots, n$ and to include at least two functions with different $\log$ derivatives at $q=q_{\mathrm{r}}$ and two functions with different $\log$ derivatives at $q=q_{p}$. The importance of this second constraint cannot be overemphasised, as it ensures that the basis is capable of representing arbitrary (outgoing-wave) log derivatives correctly at the two boundaries [17]. A simple example of a basis set which satisfies both constraints is provided by the normalised Legendre polynomials

$\phi_{i}(q)=N_{i} P_{i-1}(x)$,

where $N_{i}=\sqrt{(2 i-1) /\left(q_{\mathrm{p}}-q_{\mathrm{r}}\right)}$ for $i=1,2, \ldots, n \geqslant 2$ and $x=2\left(q-q_{\mathrm{r}}\right) /\left(q_{\mathrm{p}}-q_{\mathrm{r}}\right)-1$.

Our aim now is to construct matrix elements of the operators $\mathscr{L}_{\mathrm{r}}, \mathscr{L}_{\mathrm{p}}$ and $G^{+}(E)$ in this basis. Matrix elements of the first two of these operators are easily obtained from their definitions in eqs. (14) and (15), and are given by

$$
\left\langle i\left|\mathscr{L}_{\mathrm{r}}\right| j\right\rangle=+\frac{\hbar^{2}}{2 m} \phi_{i}\left(q_{\mathrm{r}}\right) \operatorname{Im}\left[L_{\mathrm{r}}^{+}\left(q_{\mathrm{r}}\right)\right] \phi_{j}\left(q_{\mathrm{r}}\right),
$$

and

$\left\langle i\left|\mathscr{L}_{\mathrm{p}}\right| j\right\rangle=-\frac{\hbar^{2}}{2 m} \phi_{i}\left(q_{\mathrm{p}}\right) \operatorname{Im}\left[L_{\mathrm{p}}^{+}\left(q_{\mathrm{p}}\right)\right] \phi_{j}\left(q_{\mathrm{p}}\right)$.

Matrix elements of $G^{+}(E)$ are given variationally by eq. (13) as [18]

$$
\left\langle i\left|G^{+}(E)\right| j\right\rangle=\langle i|E-\mathscr{L}(E)-H| j\rangle^{-1},
$$


where $\langle i|E-\mathscr{L}(E)-H| j\rangle^{-1}$ means the $i j$ element of the inverse of the $n \times n$ complex symmetric matrix with elements $\langle i|E-\mathscr{L}(E)-H| j\rangle$. These latter matrix elements are given by eqs. (5), (9) and (21) as

$$
\langle i|E-\mathscr{L}(E)-H| j\rangle=E \delta_{i j}-L_{i j}-H_{i j},
$$

where

$$
\begin{gathered}
L_{i j}=\frac{\hbar^{2}}{2 m}\left[\phi_{i}\left(q_{\mathrm{r}}\right) L_{\mathrm{r}}^{+}\left(q_{\mathrm{r}}\right) \phi_{j}\left(q_{\mathrm{r}}\right)\right. \\
\left.-\phi_{i}\left(q_{\mathrm{p}}\right) L_{\mathrm{p}}^{+}\left(q_{\mathrm{p}}\right) \phi_{j}\left(q_{\mathrm{p}}\right)\right],
\end{gathered}
$$

and

$H_{i j}=\int_{q_{\mathrm{T}}}^{q_{\mathrm{T}}}\left[\frac{\hbar^{2}}{2 m} \phi_{i}^{\prime}(q) \phi_{j}^{\prime}(q)+\phi_{i}(q) V(q) \phi_{j}(q)\right] \mathrm{d} q$.

Finally, using these equations, the trace in eq. (12) can be evaluated in the basis $\left\{\phi_{i}(q)\right\}_{1}^{n}$ as

$$
\begin{aligned}
& N(E)=-4\left(\frac{\hbar^{2}}{2 m}\right)^{2} \operatorname{Im}\left[L_{\mathrm{p}}^{+}\left(q_{\mathrm{p}}\right)\right] \\
& \quad \times\left|\left\langle q_{\mathrm{p}}\left|G^{+}(E)\right| q_{\mathrm{r}}\right\rangle\right|^{2} \operatorname{Im}\left[L_{\mathrm{r}}^{+}\left(q_{\mathrm{r}}\right)\right],
\end{aligned}
$$

where

$$
\begin{aligned}
& \left\langle q_{\mathrm{p}}\left|G^{+}(E)\right| q_{\mathrm{r}}\right\rangle=\sum_{i j} \phi_{i}\left(q_{\mathrm{p}}\right) \\
& \quad \times\langle i|E-\mathscr{L}(E)-H| j\rangle^{-1} \phi_{j}\left(q_{\mathrm{r}}\right),
\end{aligned}
$$

which only requires the solution of a single $n \times n$ system of complex linear equations.

Eq. (29) can be derived more directly from the expression for the scattering matrix that is given by the general theory of the log derivative version of the Kohn variation principle [19], which is also immediately applicable to more difficult multidimensional problems. However the present derivation provides a more interest connection wit the conventional use of absorbing potential boundary conditions [11,12].

\section{Example calculations}

In order to examine the properties of the absorbing log derivative boundary conditions in eqs. (19) and $(20)$ we shall now consider two simple barrier penetration problems. The first is the Eckart barrier problem shown in fig. 1 , which corresponds to the interaction potential

$V(q)=V_{0} \operatorname{sech}^{2}(q / a)$.

This is a well established model problem for which the exact barrier transmission probability $N(E)$ is known analytically $[20]$. We shall take the parameters here to be $V_{0}=0.425 \mathrm{eV}$ and $m=1060 \mathrm{au}$, which correspond to the collinear barrier in the $\mathrm{H}+\mathrm{H}_{2}$ reaction. These same parameters were also used by Seideman and Miller and allow a direct comparison with their absorbing potential results [11].

Fig. 2 shows the percent error in the calculated barrier transmission probability $N(E)$ for this prob-

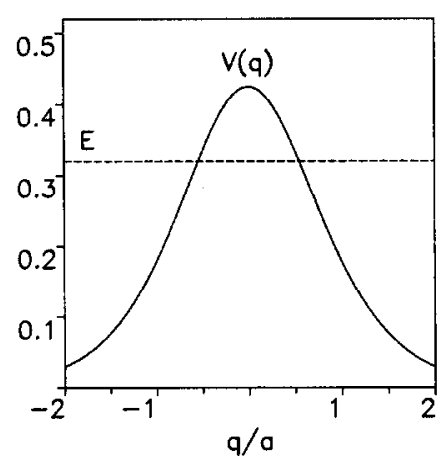

Fig. 1. The Eckart potential of eq. (31). The interaction region shown in this figure is sufficient to converge $N(E)$ to better than half a percent when absorbing log derivative boundary conditions are used at the two boundaries.

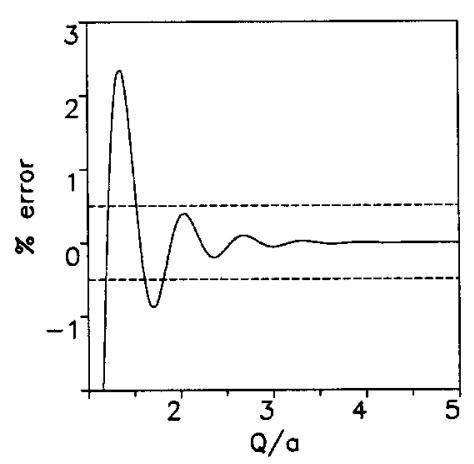

Fig. 2. Convergence of first-order WKB absorbing log derivative boundary conditions for the Eckart barrier problem as a function of the size of the interaction region $-Q \leqslant q \leqslant Q$. The exact barrier transmission probability if $N(E)=9.5110 \times 10^{-3}$ at $E=0.32 \mathrm{eV}$. 
lem at $E=0.32 \mathrm{eV}$, as a function of the size of the interaction region $-Q \leqslant q \leqslant Q$. It is clear from this figure that $N(E)$ can be calculated with an error of less than half a percent by the time $Q=2 a$, which corresponds to the physical interaction region shown in fig. 1. By contrast, the best of the various functional forms for the absorbing potential $\epsilon(q)$ employed by Seideman and Miller requires $Q>6 a$ for a one percent error in $N(E)$ at the same energy [11]. Therefore, for this problem, the present absorbing $\log$ derivative boundary conditions require an interaction region that is only about one third the size of that required by typical absorbing potential boundary conditions.

A somewhat more interesting model problem is provided by the symmetric double maximum barrier shown in fig. 3 , which corresponds to the interaction potential

$V(q)=V_{0}\left[\frac{1}{2}+(q / a)^{2}\right] \operatorname{sech}^{2}(q / a)$.

The barrier transmission probability $N(E)$ for this potential is not available analytically, but it is easily obtained numerically using eq. (29). In what follows we shall use the same parameters $V_{0}=0.425 \mathrm{eV}$ and $m=1060$ au as above, and focus on an energy $E=0.25 \mathrm{eV}$ which lies below the degenerate potential maxima but above the enclosed potential minimum.

Fig. 4 shows the percent error in the barrier transmission probability $N(E)$ for this problem, again calculated as a function of the size of the interaction region $-Q \leqslant q \leqslant+Q$. It is clear from this figure that

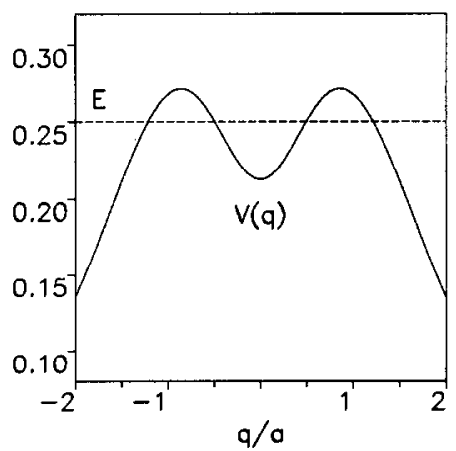

Fig. 3. The symmetric double maximum potential of eq. (32). The interaction region shown in this figure is again sufficient to converge $N(E)$ to better than half a percent when absorbing log derivative boundary conditions are used at the two boundaries.

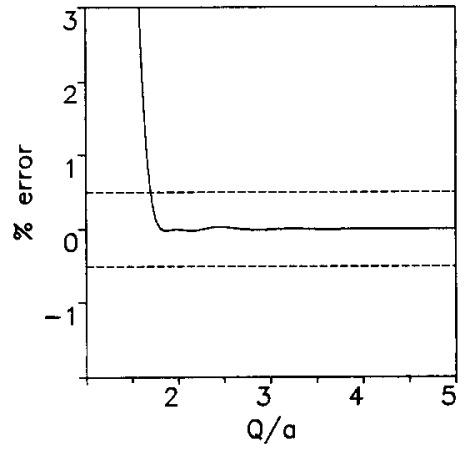

Fig. 4. Convergence of first-order WKB absorbing log derivative boundary conditions for the symmetric double maximum barrier problem as a function of the size of the interaction region $-Q \leqslant q \leqslant Q$. The exact barrier transmission probability is $N(E)=0.99980$ at $E=0.25 \mathrm{eV}$.

$N(E)$ can again be calculated to within half a percent by applying absorbing log derivative boundary conditions at $q_{\mathrm{r}}=-2 a$ and $q_{\mathrm{p}}=+2 a$, which correspond to the boundaries of the physical interaction region shown in fig. 3. This is perhaps an even more impressive demonstration of the present absorbing log derivative boundary conditions than the Eckart barrier problem considered above, because the double maximum interaction potential $V(q)$ and its first derivative $V^{\prime}(q)$ are still very significant at these two boundaries. Indeed the boundaries are already very close to the outer classical turning points at $E=0.25$ $\mathrm{eV}$ in fig. 3, which provide an absolute lower bound on the size of the physical interaction that can reasonably be used for calculating $N(E)$ at this energy.

Fig. 5 shows that this rapid convergence is not confined to a single energy, but is also obtained throughout the range $E=0.22$ to $0.34 \mathrm{eV}$ which extends to above the barrier maxima. In fact the convergence with respect to the required size of the interaction region is found to be even better at these higher energies because the semiclassical absorbing boundary conditions in eqs. (19) and (20) become more accurate. This is especially interesting because a purely quantum mechanical calculation usually requires more work at higher energies. The present combination of a quantum mechanical description of the transition state region with semiclassical absorbing boundary conditions might generally be expected to scale better.

It can also be seen from fig. 5 that the exact barrier 


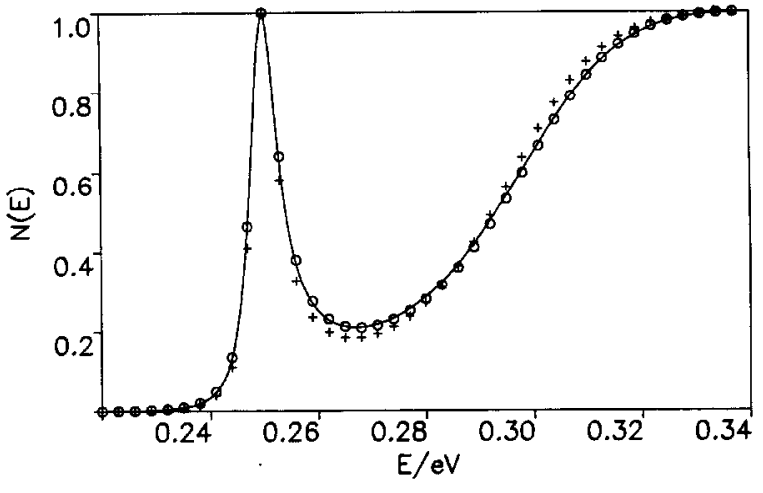

Fig. 5. The barrier transmission probability $N(E)$ versus $E$ for the symmetric double maximum potential. The solid line is the converged quantum mechanical result. The crosses (and circles) are obtained when zero-order (and first-order) WKB absorbing $\log$ derivative boundary conditions are applied at $q= \pm 2 a$. The pronounced peak in $N(E)$ close to $E=0.25 \mathrm{eV}$ is an example of resonant tunnelling (see text).

transmission probability $N(E)$ for the symmetric double maximum potential problem exhibits a pronounced "perfect tunnelling" peak $(N(E)=1)$ at an energy $E \approx 0.25 \mathrm{eV}$ below the degenerate potential maxima. This curious result is due to the phenomenon of resonant tunnelling, which is to say that the barrier becomes completely transparent at the energy of a quasi-bound state associated with its well region even in the limit of deep tunnelling. A semiclassical analysis that applies the connection formulae described by Child [15] to the symmetric double maximum potential problem explains this phenomenon very clearly (see also the discussion by Bohm [21]). In the sharp resonance limit, in which the energy lies well below the degenerate barrier maxima, the Siegert [22] resonance condition of purely outgoing waves as $q \rightarrow \pm \infty$ is found to give a barrier resonance energy $E=E_{v}-\frac{1}{2} \mathrm{i} \Gamma_{\nu}$ with a real part $E_{\nu}$ which corresponds exactly to the perfect tunnelling condition $N\left(E_{v}\right)=1$.

The behaviour seen in fig. 5 is simply a (purely quantum mechanical) manifestation of this resonant tunnelling effect for the present $v=0$ barrier resonance, which corresponds to the Siegert eigenvalue $E=0.24939-0.00312 \mathrm{i} \mathrm{eV}$. (A resonance eigenvalue $E=E_{v}-\frac{1}{2} \Gamma_{v}$ occurs at a pole of the scattering Green function $G^{+}(E)$, and can therefore be calculated within the present formulation as a complex root of the equation

$\operatorname{det}[\langle i|E-\mathscr{L}(E)-H| j\rangle]=0$,

where $\operatorname{det}[\langle i|A| j\rangle]$ denotes the determinant of the $n \times n$ matrix with elements $\langle i|A| j\rangle$.) Resonant tunnelling was first observed experimentally in the conductance of GaAlAs double barriers [23], and it continues to play an important role in the theory of semiconductor devices [24]. It is conceivable that it might also play a role in enhancing the rates of chemical and charge transfer reactions.

\section{Conclusion}

In this Letter, we have shown that the reaction rate theory of Miller, Schwartz and Tromp can be reformulated in such a way that the scattering boundary conditions are applied by a complex Bloch boundary value operator at the boundary of a finite interaction region. The outgoing-wave log derivatives that appear in this Bloch operator can be approximated semiclassically, resulting in a genuine quantum mechanical transition region theory of reaction rates [4] (i.e., a theory involving a quantum mechanical description of the transition state or interaction region in conjunction with approximate semiclassical absorbing boundary conditions ). The theory also eventually converges on what is essentially the exact quantum mechanical result as the size of the interaction region is increased, because the semiclassical boundary conditions become exact as the derivatives of the interaction potential tend to zero. Indeed example applications to model barrier penetration problems have shown that this convergence with respect to the required size of the interaction region can be very rapid, and this is what gives the theory its appeal.

Perhaps the most obvious explanation for why the present absorbing log derivative boundary conditions work so well is that they are explicitly energydependent, just like the exact outgoing-wave scattering boundary conditions they approximate. This is in sharp contrast to the more familiar use of energy-independent absorbing potentials (compare eqs. (7) and (8)). Moreover the present absorbing boundary conditions are clearly exact within the first- 
order WKB approximation that was used to derive them, rather than simply $99 \%$ [25] or $99.9 \%$ [11] accurate within the same approximation in the special case of a constant interaction potential at the boundary.

The present theory has only been developed in detail for the simple case of one-dimensional barrier crossing, but appropriate generalisations are available throughout the argument $[13,16,19]$. It might therefore be hoped that some combination of these generalisations with the various other interesting ideas described by Seideman and Miller (including in particular their appealingly general suggestion of using a discrete variable representation of the Watson Hamiltonian referenced at the transition state [12]) will eventually lead to an efficient scheme for the calculation of quantum mechanical cumulative reaction probabilities and thermal rate constants even for polyatomic chemical reactions.

\section{Acknowledgement}

The work described in this Letter is based on a conversation between the authors at the 14th Conference on the Dynamics of Molecular Collisions, Helen, GA, USA (6-11 June 1993). One of the authors (JCL) acknowledges partial support for this research by the Department of Energy under grant DE-FG02-87ER13697.

\section{References}

[1] W.H. Miller, J. Chem. Phys. 62 (1975) 1899.
[2] D.G. Truhlar and B.C. Garrett, Annu. Rev. Phys. Chem. 35 (1984) 159.

[3] W.H. Miller, Faraday Discussions Chem. Soc. 62 (1977) 40.

[4] W.H. Miller, Accounts Chem. Res. 26 (1993) 174.

[5] W.H. Miller, S.D. Schwartz and J.W. Tromp, J. Chem. Phys. 79 (1983) 4889.

[6] J.W. Tromp and W.H. Miller, Faraday Discussions Chem. Soc. 84 (1987) 441.

[7] T.P. Park and J.C. Light, J. Chem. Phys. 91 (1989) 974

[8] T.P. Park and J.C. Light, J. Chem. Phys. 94 (1991) 2946.

[9] P.N. Day and D.G. Truhlar, J. Chem. Phys. 94 (1991) 2045.

[10] D. Neuhauser and M. Baer, J. Chem. Phys. 90 (1989) 4351.

[11] T. Seideman and W.H. Miller, J. Chem. Phys. 96 (1992) 4412.

[12] T. Seideman and W.H. Miller, J. Chem. Phys. 97 (1992) 2499.

[13] C. Bloch, Nucl. Phys. 4 (1957) 503.

[14] A.M. Lane and R.G. Thomas, Rev. Mod. Phys. 30 (1958) 257.

[15] M.S. Child, Semiclassical mechanics with molecular applications (Oxford Univ. Press, Oxford, 1991) ch. $2,3$.

[16] B.R. Johnson, Chem. Phys. 2 (1973) 831.

[17] R.K. Nesbet, Variational methods in electron-atom scattering theory (Plenum, New York, 1980) p. 53.

[18] W.H. Miller and B.M.D.D. Jansen op de Haar, J. Chem. Phys. 86 (1987) 6213.

[19] D.E. Manolopoulos, M. D'Mello and R.E. Wyatt, J. Chem. Phys. 91 (1989) 6096.

[20] H.S. Johnston, Gas phase reaction rate theory (Ronald, New York, 1966) pp. 37-47.

[21] D. Bohm, Quantum theory (Prentice-Hall, Englewood Cliffs, 1951) pp. 283-295.

[22] A.J.F. Siegert, Phys. Rev. 56 (1939) 750.

[23] L.L. Chang, L. Esaki and R. Tsu, Appl. Phys. Letters 24 (1974) 593.

[24] S. Wang, Fundamentals of semiconductor theory and device physics, section 13.3 (Prentice-Hall, Englewood Cliffs, 1989).

[25] M.S. Child, Mol. Phys. 72 (1991) 89. 